\title{
Three sternalis muscles associated with abnormal attachments of the pectoralis major muscle
}

\author{
Levent Sarıkçığlu ${ }^{1}$, Bahadır Murat Demirel ${ }^{1}$, Nurettin Oğuz ${ }^{1}$, Yaşar Uçar ${ }^{1}$ \\ ${ }^{1}$ Department of Anatomy, Akdeniz University Faculty of Medicine, Antalya, Turkey
}

\begin{abstract}
During dissection of the thoracoabdominal region of a 56-year-old male cadaver, we encountered three sternalis muscles (one on the right side, two on the left side) covered by superficial fascia and located anterior to the pectoralis major muscle. Additionally, abnormal attachment of the pectoralis major muscle was also observed. We think that the existence of the sternalis muscle and abnormal attachment of the pectoralis major muscle should not be overlooked in the surgical and diagnostic procedures.
\end{abstract}

Key words: sternalis muscle; pectoralis major muscle; cadaver.

Anatomy 2008; 2: 67-71, (c) 2008 TSACA

\section{Introduction}

The sternalis muscle is an uncommon anatomic variation of chest wall musculature. According to Turner, Cabrolius was the first anatomist to observe the sternalis muscle in 1604. ${ }^{1}$ Although the sternalis muscle has been encountered in the 17 th century, recently many articles have also been reported on the sternalis muscle..$^{2-8}$

The sternalis muscle is called a normal anatomic variant and a well-known variation although in most anatomical textbooks it is insufficiently mentioned. Although the sternalis muscle has been well described in the literature, some confusion persists. For instance, it is presented in Gray's Anatomy as a variation of pectoralis major and is called rectus sternalis, whereas in Larsen's Human Embryology it is presented as a derivative of the rectus column and called sternalis muscle..$^{9-10}$

The sternalis muscle is often bilateral and sometimes associated with the variation of the pectoralis major muscle. ${ }^{11-13}$ We report here an example of appearance of the sternalis muscles with accompanying pectoralis major muscle variation and review the innervation and homology of the sternalis muscle.

\section{Case Report}

During dissection of the thoracoabdominal region of a 56-year-old male cadaver, we encountered three sternalis muscles (one on the right side, two on the left side) covered by superficial fascia and located anterior to the pectoralis major muscle.

First sternalis muscle (SM1) was found on the right side. It originated from the sheath of the right external oblique muscle, sixth and seventh costal cartilages. It run upward almost parallel to the sternum and attached to the left sternalis muscle (SM2) about $2 \mathrm{~cm}$ inferior to the sternal angle (angle of Louis) and $7 \mathrm{~cm}$ inferior to the jugular notch (Figure 1a). Innervation of the muscle was from the anterior cutaneous branch of the sixth intercostal nerve (Figure 1b). 


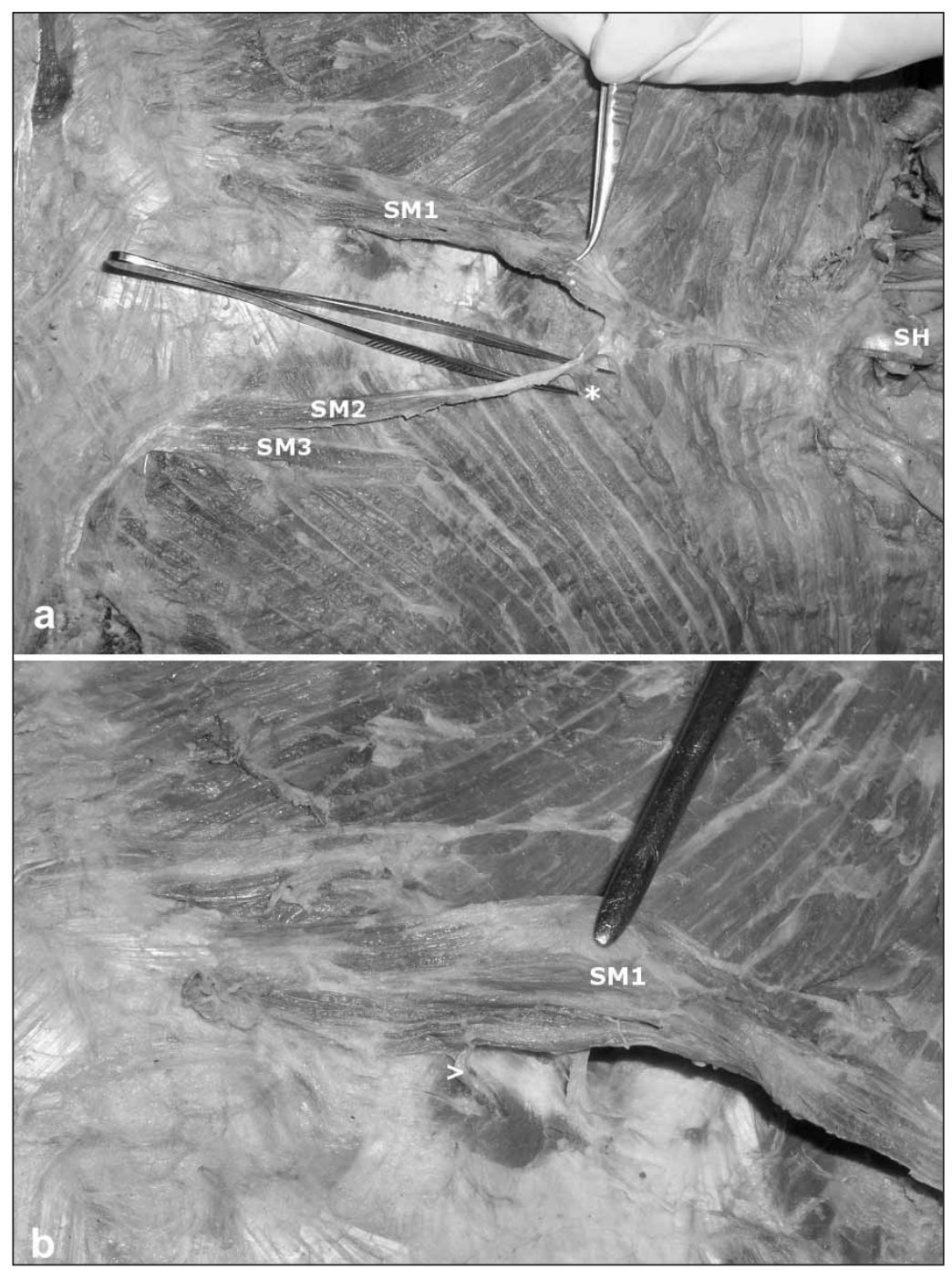

Figure 1. Photograph of the pectoral region of the case. (a) Three sternalis muscles and their attachments. (b) Innervation of the right sternalis muscle. SH: sternal head of the left sternocleidomastoid muscle; SM1: first sternalis muscle; SM2: second sternalis muscle; SM3: third sternalis muscle; *: Fibers of the pectoralis major muscle attaching to the tendon of the second sternalis muscle; arrowhead: twig of anterior cutaneous branch of the sixth intercostal nerve.

Second sternalis muscle (SM2) was located on the left side. It originated from the sheath of the external oblique muscle and ran upward. Some fibers of the sternocostal part of the left pectoralis major muscle were also attached to the tendon of the SM2 (Figure 1). A fanshaped tendon of this muscle attached to the body of the sternum and to the SM1 to form a common tendon. The common tendon of SM1 and SM2 then attached to the sternal head of the right sternocleidomastoid muscle. Furthermore, before the common tendon attached to the sternal head of the right sternocleidomastoid muscle, some fibers of the sternocostal part of the pectoralis major muscle attached to common tendon of the sternalis muscles (Figures $2 \mathbf{a}$ and $\mathbf{2 b}$ ). Innervation of the second sternalis muscle was also from the anterior cutaneous branch of the sixth intercostal nerve. The intercostal nerve entered into the sternalis muscle from medial to lateral direction (Figure 2c).

Third sternalis muscle (SM3) was a fleshy band and observed in the left side. It originated from the same area where the SM2 originated. It ran upward, laterally, and finally fused to the fibers of the left pectoralis major muscle (Figure 2c). The innervation source of this muscle could not be traced.

\section{Discussion}

The sternalis muscle is a fleshy band of longitudinal fibers of varying length and width, located close to and 
Figure 2. Close view of the pectoral region of the case. (a) Three sternalis muscles and their attachments. (b) Common tendon of the sternalis muscle attaching to the sternal head of the sternocleidomastoid muscle. (c) Innervation of two sternalis muscles. CT: common tendon of the sternalis muscles; PM: fibers of the pectoralis major muscle attaching to the common tendon of the sternalis muscles; SM1: first sternalis muscle; SM2: second sternalis muscle; SM3: third sternalis muscle; arrowheads: twig of anterior cutaneous branch of the sixth intercostal nerve.
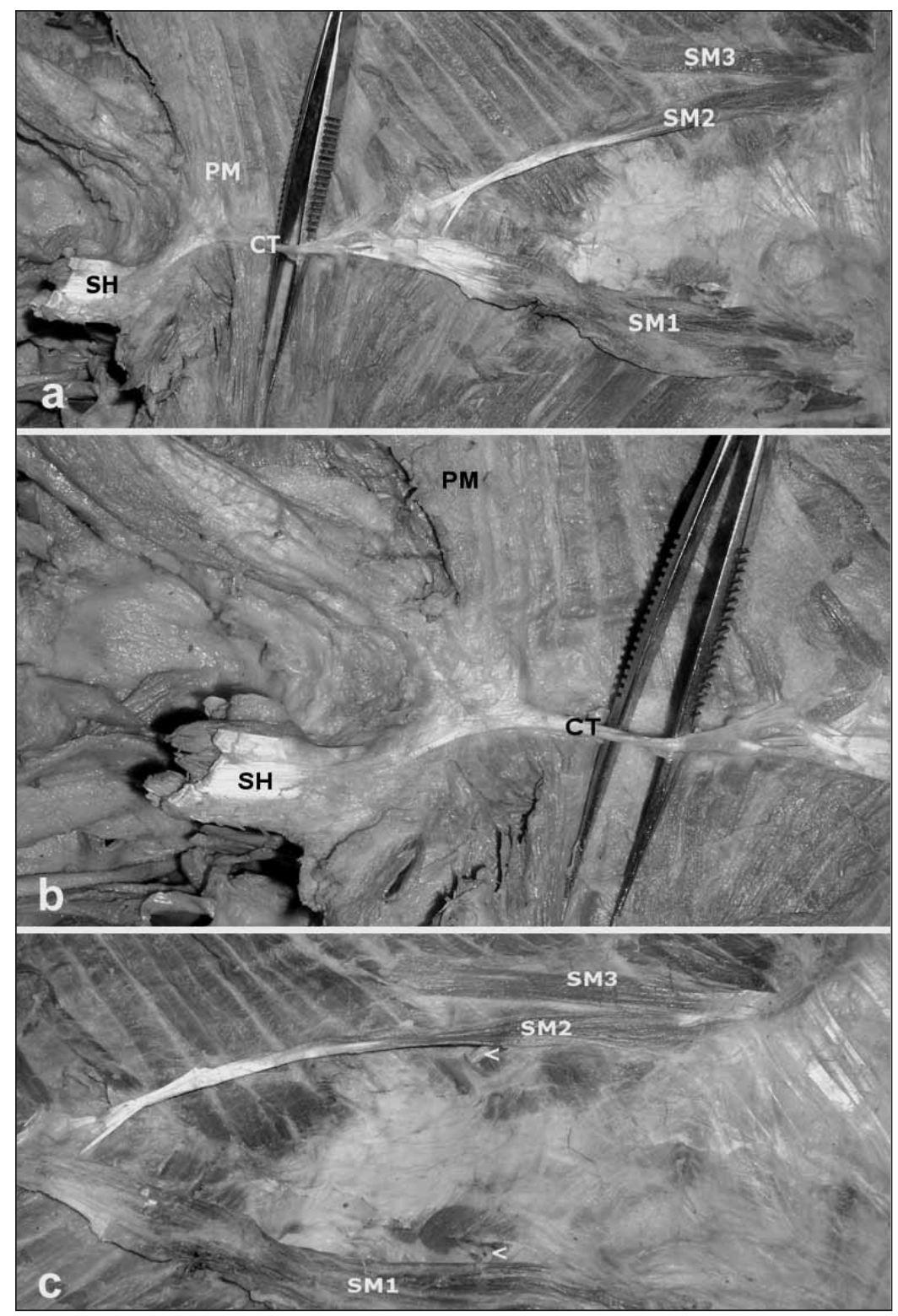

generally parallel to the sternum. When typical, it arises from the sheath of the rectus abdominis muscle, aponeurosis of the external oblique muscle, pectoralis major muscle, or costal cartilages and ends above onto the upper costal cartilages, manubrium, or may be joined to the sternal head of the sternocleidomastoid muscle. ${ }^{9}$ In the present case, the common tendon of the two sternalis muscles attached to the sternal head of the left sternocleidomastoid muscle.

The incidence of the sternalis muscle varies widely between nationalities. Its reported incidence is $4 \%$ to
$7 \%$ in the white population, $8.4 \%$ in the black population, and $11.5 \%$ in the Asian population. ${ }^{11}$ In some populations such as Taiwanese, the incidence may be as low as $1 \% .^{14-15}$ In Turkish population, the incidence was reported as $9.3 \% .{ }^{16}$ When the sternalis muscle accompanies partial or complete congenital absence of the pectoralis major muscle, it may achieve significant size..$^{17}$

Although the sternalis muscle has been encountered since its first description in the 17 th century, the derivation and nerve supply of the sternalis muscle have been a conundrum for many anatomists. Many authors assumed 
the sternalis muscle to be derived from neighboring muscles, such as pectoralis major muscle, ${ }^{12,18-19}$ rectus abdominis muscle, ${ }^{20-21}$ sternocleidomastoid muscle, ${ }^{20-21}$ panniculus carnosus, ${ }^{15,22}$ and external oblique muscle. ${ }^{23}$ According to Turner, it is presented in man as a result of atavism of the fascicles of the pectoralis cutaneous muscle of lower animals. ${ }^{1}$ Sadler described it as a part of a ventral, longitudinal column of muscle arising at the ventral tips of the hypomeres. ${ }^{24}$ Ruge considered the sternalis muscle to represent a vestige of the cuticular muscle of mammals that constitutes the great subcutaneous muscle of the trunk; it also presents in man in the form of the axillary arch. ${ }^{18}$ Barlow claims that the sternalis muscle represents the remains of the panniculus carnosus. ${ }^{22}$ In the present case, three sternalis muscles were found to be covered by superficial fascia of the anterior thoracic region. For that reason, a derivation of the sternalis muscle from the panniculus carnosus cannot be excluded.

Majority of sternalis muscle are supplied by intercostal nerves. ${ }^{13-15,20-22,25}$ Additionally, participation of the pectoral nerves has also been reported. ${ }^{26}$ O'Neill and Folan-Curran reviewed the literature and found that $55 \%$ of the sternalis muscles were innervated by branches of the internal and external thoracic nerves, $43 \%$ by branches of the intercostal nerves and $2 \%$ both from the intercostal and thoracic nerves. ${ }^{13}$ In their case, the sternalis muscle was innervated by the intercostal nerve. The question of the double innervation of the sternalis muscle was addressed by Kida and Kudoh. ${ }^{12}$ Kida et al. reported some difficulties for finding the innervation source of the sternalis muscle. ${ }^{26}$ They suggest that it is difficult to find the twigs from the pectoral nerves supplying the sternalis muscle since when the lateral rim of the sternalis muscle has been clearly dissected, the very fine twigs, easily confused with the connective tissue, are almost always removed or damaged. In the present case, two sternalis muscles were innervated by the intercostal nerves. The intercostal nerves entered into the sternalis muscles from medial to lateral direction. Additionally, the innervation source of the third sternalis muscle could not be traced due to small size of the muscle and may be due to some difficulties reported by Kida et al. ${ }^{26}$
O'Neill and Folan-Curran reported the direct connection of muscle fibers between the sternalis and pectoralis major muscles. ${ }^{13}$ Additionally, Kida et al. reported the partial absence of the sternocostal portion of the pectoralis major muscle. ${ }^{26}$ They suggested that the close relation of both muscles might show their origin, although a connection of muscle fibers did not always indicate a muscle origin. They supported the opinion of the sternalis muscle derivation from the pectoralis major muscle.

Shinohara reported three laws (migration, fusion and separation) on nerve-muscle specificity. ${ }^{27}$ In the law of migration, the nerve is regarded as an indicator of the route along which the muscle mass originated and migrated (i.e. diaphragm). In the law of fusion, when a muscle is supplied by two different nerves, this muscle is considered to have been formed by fusion of two muscle mass, each of which was originally supplied by a separate nerve. Some muscle, e.g. the external oblique, are supplied both from the superficial and deep surfaces, and this dual surface supply is interpreted as suggesting that the muscle is a composite muscle. According to the law of separation, two different muscles supplied by a single nerve are considered to be derived from a single muscle mass (i.e. common muscle mass for the sternecleidomastoid and trapezius muscles). We think that further studies should be performed to clarify the derivation of the sternalis muscle on the view of these laws.

Sternalis is not only a rare muscle variant; it may have important clinical significance. The sternalis muscle is occasionally imaged on mammography, magnetic resonance imaging, or computerized tomographic scans. ${ }^{3,28-29}$ During routine mammography, sternalis muscle may be visible as an irregular structure medially on the craniocaudal projection. It may be seen in the medial aspect of the breast on the craniocaudal view as a density with an ill-defined margin and can give rise to diagnostic confusion with a breast cancer. ${ }^{28}$ Four cases were encountered among approximately 32.000 mammograms, stimulated by an index case who underwent open surgical biopsy for a presumed breast mass, actually a sternalis muscle. It is interesting to note that the frequency of sternalis muscle $(\sim 0.01)$ established by these authors is very different from the sectional frequency of woman given by Barlow. ${ }^{22}$ The rarity of sternalis muscle in the above radi- 
ological investigation could be explained not only by the more common unilateral occurrence of this muscle so that it is often unrecognizable on mammography, but also by a possibly real lower frequency in females. ${ }^{22,30} \mathrm{We}$ think that the existence of such complex arrangement of the sternalis muscle as in our case should not be overlooked in the surgical and diagnostic procedures.

\section{Acknowledgments}

We thank Mr. O. Kuru, N. Sağıroğlu, H. Gezer and H. Savc1l for their technical assistance.

\section{References}

1. Turner WM. On the musculus sternalis. 7 Anat Physiol 1867; 1: 246-53.

2. Arraez-Aybar LA, Sobrado-Perez J, Merida-Velasco JR. Left musculus sternalis. Clin Anat 2003; 16: 350-4.

3. Cawson JN, Papadopoulos T. Variants of sternal insertion of the pectoral muscle on mammography: a pictorial review. Clin Radiol 2002; 57: 442-8.

4. Harish K, Gopinath KS. Sternalis muscle: importance in surgery of the breast. Surg Radiol Anat 2003; 25: 311-4.

5. Kumar H, Rath G, Sharma M, Kohli M, Rani B. Bilateral sternalis with unusual left-sided presentation: a clinical perspective. Yonsei Med f 2003; 44: 719-22.

6. Loukas M, Bowers M, Hullett J. Sternalis muscle: a mystery still. Folia Morphol (Warsz) 2004; 63: 147-9.

7. Saeed M, Murshid KR, Rufai AA, Elsayed SE, Sadiq MS. Sternalis. An anatomic variant of chest wall musculature. Saudi Med $\mathcal{F} 2002$; 23: 1214-21.

8. Scott-Conner CE, Al-Jurf AS. The sternalis muscle. Clin Anat 2002; 15: 67-9.

9. Salmons S. Gray's Anatomy. In: Williams PL, Bannister LH, Berry $\mathrm{M}$ et al., eds. New York: Churchill-Livingstone; 1995. p. 838.

10. Larsen WJ (ed). Human Embryology. ed. Edinburgh: ChurchillLivingstone; 1997.

11. Bergman RA, Thompson SA, Afifi AK, Saadeh FA. Compendium of Human Anatomic Variation. Baltimore: Urban \& Schwarzenberg; 1988.
12. Kida MY, Kudoh H. Innervation of the sternalis muscle accompanied by congenital partial absence of the pectoralis major muscle. Okajimas Folia Anat 7pn 1991; 67: 449-55.

13. O'Neill MN, Folan-Curran J. Case report: bilateral sternalis muscles with a bilateral pectoralis major anomaly. 7 Anat 1998; 193 (Pt 2): 289-92.

14. Jeng H, Su SJ. The sternalis muscle: an uncommon anatomical variant among Taiwanese. 7 Anat 1998; 193 ( Pt 2): 287-8.

15. Shen CL, Chien CH, Lee SH. A Taiwanese with a pair of sternalis muscles. Kaibogaku Zasshi 1992; 67: 652-654.

16. Demir S, Oguz N, Sarikcioglu L, Ozkan O. M. sternalis. Morfoloji Dergisi 1999; 6: 53-6.

17. Kitamura S, Yoshioka T, Kaneda M, Matsuoka K, Chen KL, Sakai A. A case of the congenital partial defect of the pectoralis major-accompanied by the sternalis with enormous size. Kaibogaku Zasshi 1985; 60: 728-32.

18. Ruge G. Der Hautrumpfmuskel der Saugetiere:der M. sternalis und der Achselbogen des Menschen. Morph Fabrb 1905; 33: 379-531.

19. Yap SE. Musculus sternalis in Filipinos. Anat Rec 1921; 21: 353-71.

20. Blees G. A peculiar type of sternalis muscle. Acta Morphol Neerl Scand 1968; 7: 69-72.

21. Rao VS, Rao GRKH. The sternalis muscle. 7 Anat Soc India 1954; 3: 49-51.

22. Barlow RN. The sternalis muscle in American whites and Negroes. Anat Rec 1935; 64: 413-26.

23. Testus L. Les Anomalies Musculaires chez l'Homme. ed. Paris: Masson; 1884.

24. Sadler TW. Langman Medical Embryology. 7th ed. Baltimore: Williams \& Wilkins; 1995.

25. Misra BD. The sternalis muscle. J Anat Soc India 1954; 3: 47-8.

26. Kida MY, Izumi A, Tanaka S. Sternalis muscle: topic for debate. Clin Anat 2000; 13: 138-40.

27. Shinohara H. A warning against revival of the classic tenets of gross anatomy related to nerve-muscle specificity. F Anat 1996; 188 ( Pt 1): 247-8.

28. Bradley FM, Hoover HC, Jr., Hulka CA, et al. The sternalis muscle: an unusual normal finding seen on mammography. $A 7 R A m \mathcal{F}$ Roentgenol 1996; 166: 33-6.

29. Murphy JS, Nokes SR. Radiological case of the month. Sternalis muscle. 7 Ark Med Soc 1996; 93: 55-6.

30. Bailey PM, Tzarnas CD. The sternalis muscle: a normal finding encountered during breast surgery. Plast Reconstr Surg 1999; 103: 1189-90.

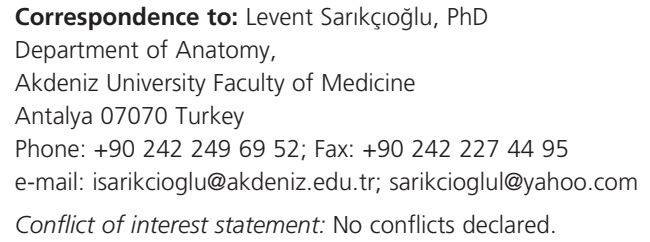

